\title{
Phénoconversion et transduction mécanogénique du coeur de mammifère
}

L'hypertrophie cardiaque secondaire à une surcharge du travail hémodynamique s'accompagne de modifications fonctionnelles permettant au cœur de s'adapter, dans une première phase, aux conditions nouvelles. Les modifications de l'expression des gènes à l'origine de ces phénomènes commencent à être connues : (1) de nouvelles isoformes de myosine et $d$ 'actine sont synthétisées par activation des gènes correspondants ; (2) l'expression du gène codant pour la $\mathrm{Ca}^{++}$-ATPase du réticulum sarcoplasmique ne change pas, ce qui aboutit à une diminution de la densité de cette enzyme clé de la relaxation des fibres cardiaques ; (3) le gène du facteur auriculaire natriurétique est stimulé dans le ventricule, aboutissant à une production accrue de cette hormone qui contribue à améliorer les conditions de charge du muscle cardiaque. A noter que beaucoup de ces changements rappellent le phénotype du cœur fœtal. La nature des messagers informant les systèmes de contrôle génique de l'étirement des fibres musculaires reste mal connue. L'AMP cyclique et des changements de concentration ionique, provoqués par la stimulation de canaux spécifiques, pourraient être en cause.

Ketty Schwartz

\section{ADRESSE} 664
K. Schwartz : directeur de recherche. Inserm

K. Schwartz : directeur de recherche. Inserm demande fonctionnelle. Depuis le début de ce siècle, trois approches orsque, il y a environ 300 ans, sir William Harvey décrivit le principe de la circulation sanguine, il observa également que le débit sanguin pouvait être modifié par divers facteurs, externes ou internes, naturels ou non, tels que le sommeil, le repos, la nourriture, l'exercice et même les mouvements de l'âme. En fait, ce médecin génial avait déjà découvert que la propriété la plus fondamentale du myocarde était de s'adapter à une nouvelle distinctes ont successivement expliqué des aspects complémentaires de la régulation du travail cardiaque [1] (Tableau I). La première, biophysique, était centrée sur le rôle de la longueur télédiastolique de la fibre musculaire et pouvait rendre compte de réponses immédiates à des altérations de pré- ou de post-charge, par exemple régulation de battement à battement ou égalisation des débits des deux ventricules. Ce fut la loi de Starling (1912). La deuxième, biochimique, expliqua le mode d'action de diverses drogues et neurotransmetteurs - celui, par exemple, des ago- 
nistes $\beta$-adrénergiques sur le myocyte et la régulation de la contractilité cardiaque.

Le propos de cet article est de détailler la troisième approche, que l'on pourrait qualifier de génétique, qui permet de comprendre comment le cœur peut s'autocontrôler en modifiant sa propre expression génique. L'importance de cette approche tient à ce qu'elle permet d'expliquer certaines des altérations des performances contractiles du myocarde soumis aux surcharges chroniques de travail hémodynamique qui sont partie intégrante de la majorité des atteintes cardiovasculaires, première cause de mortalité et de morbidité dans nos pays occidentaux. Ces surcharges produisent toutes une hypertrophie du tissu cardiaque, évoluant schématiquement en trois étapes, la phase précoce d'installation de l'hypertrophie, la phase d'hypertrophie compensée pendant laquelle le muscle cardiaque s'adapte au surcroît de travail hémodynamique, et enfin la phase décompensée au moment où survient l'insuffisance ventriculaire [2].

Les concepts de phénoconversion* et de transduction mécanogénique** prirent naissance lorsque l'on observa que le ralentissement de la contraction du cœur de rat, au stade d'hypertrophie compensée, était dû à la présence d'une isoforme de la myosine différente de l'isoforme habituelle (V3 au lieu de V1) [3, 4 et voir médecine-sciences, $n^{\circ} 2$, vol. 3, p. 66]. Depuis ce moment, le très grand pouvoir de résolution des techniques du génie génétique a permis de découvrir que l'expression d'autres gènes cardiaques était modifiée. Nous résumerons ici les données les plus récentes concernant les modifications de l'expression des gènes observées lors des altérations des trois principales fonctions du cœur hypertrophié : expression différentielle des familles multigéniques de la myosine et de l'actine dans les anomalies de la contraction, non-activation du gène codant pour la $\mathrm{Ca}^{++}-\mathrm{ATPase}$ du réticulum sarcoplasmique dans les

* Phénoconversion : modification du phénotype. ** Transduction mécanogénique: régulation de l'expression des gènes par des facteurs mécaniques. $\mathrm{m} / \mathrm{s} n^{\circ} 7$ vol. 6 , septembre 90

\section{Tableau I}

\section{LES DIVERS MÉCANISMES DE RÉGULATION DU TRAVAIL CARDIAQUE}

1 - Régulation par modification de la longueur télédiastolique de la fibre cardiaque (Loi de Sarling, 1912)

$\rightarrow$ régulation de battement à battement, ajustement des débits de deux ventricules.

2 - Régulation par changements biochimiques dans les myocytes (couplage excitation-contraction et contractilité myocardique, 1950) --) réponse à court terme à divers agents physiologiques et pharmacologiques, c'est-à-dire neurotransmetteurs et médicaments

3 - Régulation par modification de l'expression génique (1985) $\rightarrow$ réponses à long terme à des surcharges chroniques de travail hémodynamique, vieillissement et endocrinopathies.

D’après [1]

altérations de la relaxation, et enfin activation ventriculaire du gène du facteur atrial natriurétique dans les anomalies de la fonction endocrine. Des données parcellaires ont été obtenues dans plusieurs espèces animales, mais l'essentiel des connaissances concerne le rat et l'homme.

\section{Expression différentielle des familles multigéniques de la myosine et de l'actine}

La myosine et l'actine sont les constituants essentiels respectivement des filaments épais et fins du sarcomère. Ce sont les deux principaux effecteurs de la contraction cardiaque, l'énergie nécessaire étant fournie par l'activation par l'actine de l'activité ATPasique de la myosine. Alors que l'actine est une protéine monomérique globulaire, la myosine est un hexamère, formé de deux chaînes lourdes, MHC (myosin heavy chain), et de deux types de chaînes légères, MLC (myosin light chain). Ce sont les MHC qui portent les sites d'hydrolyse de l'ATP et de fixation à l'actine, et nous ne détaillerons pas ici les données concernant les chaînes légères.

Isochaînes lourdes de myosine $\mathrm{Au}$ minimum deux isochaînes lourdes, $\alpha \mathrm{MHC}$ et $\beta \mathrm{MHC}$, sont présentes dans les myocytes cardiaques [revues dans 5 et 6]. Elles sont le produit de deux gènes distincts, d'environ 24000 nucléotides contenant 41 exons. Les deux isogènes sont situés en tandem (le gène $\beta$ est en amont du gène $\alpha$ ) sur un même segment génomique de 50 kilobases chez le rat (chromosome 14) et 51 chez l'homme (chromosome 3 ) et ils sont séparés par environ respectivement 4 et 4,5 kilobases de séquences non codantes. L'homologie entre les isogènes est d'environ 20 à $30 \%$, ce qui représente une homologie de 90 à $95 \%$ entre les séquences codantes. Des différences nettes existent dans le peptide carboxyl terminal et dans les régions 3' non traduites. Les degrés d'homologie connus avec d'autres isoformes de myosine (squelettique embryonnaire, squelettique néonatale, squelettiques rapides IIA et IIB) suggèrent que la différenciation, dans une même espèce animale, entre myosines de muscles du squelette et de muscle cardiaque serait apparue avant la divergence entre oiseaux et mammifères $\left(3 \times 10^{8}\right.$ ans $)$. Les protéines $\alpha \mathrm{MHC}$ et $\beta \mathrm{MHC}$ sont associées dans les ventricules aux chaînes légères de type ventriculaire et constituent les trois isomyosines décrites en 1978: $\mathrm{V} 1=\alpha \mathrm{MHC}$, $\alpha \mathrm{MHC}, \quad \mathrm{V} 2=\alpha \mathrm{MHC}, \beta \mathrm{MHC}$, et $\mathrm{V} 3=\beta \mathrm{MHC}, \beta \mathrm{MHC}[3]$. Dans les oreillettes, $\alpha \mathrm{MHC}$ et $\beta \mathrm{MHC}$ sont associées aux chaînes légères auriculaires, mais les isomyosines correspondantes sont mal définies.

L'effet général des surcharges de travail hémodynamique, quels que 
soient le tissu ou l'espèce animale, est d'entraîner une accumulation de $\beta \mathrm{MHC}$ et une diminution parallèle de $\alpha \mathrm{MHC}$, à condition que le phénotype initial le permette [revues dans 5 et 6 et Tableau II]. Cela a d'abord été démontré dans le ventricule de rat [4], pour lequel de nombreuses études ont été conduites au cours de la dernière décennie. Comme $\beta \mathrm{MHC}$ est l'isoforme prédominante du ventricule fœtal, l'hypothèse a été émise d'une réexpression globale d'un phénotype fœtal dans le myocyte adulte hypertrophié. Le changement se produit également dans les oreillettes humaines, pour lesquelles les données sont plus récentes et moins nombreuses. Dans ce tissu, la proportion de $\alpha \mathrm{MHC}$ est nettement diminuée dans des biopsies de l'oreillette gauche réalisées au cours d'interventions de remplacement valvulaire mitral [7]. De plus, tous patients confondus, il existe une corrélation linéaire négative entre la proportion de $\alpha \mathrm{MHC}$ et la taille de l'oreillette gauche, signifiant probablement que la phénoconversion de la myosine de l'oreillette humaine est d'autant plus importante que la surcharge hémodynamique est ancienne et sévère (figure 1). Donc, dans les deux tissus où cela a été précisément déterminé, ventricule de rat et oreillette humaine, il semble exister une relation directe entre degré et intensité de surcharge de travail et synthèse de l'isoforme $\beta \mathrm{MHC}$.

Le niveau de régulation de l'expression des isogènes $\alpha \mathrm{MHC}$ et $\beta \mathrm{MHC}$ est mal connu. Dans le décours de l'hypertrophie ventriculaire gauche du rat, l'ARNm $\beta$ MHC s'accumule avant la protéine, puis ARNm et protéine se stabilisent au même niveau [8]. Des résultats préliminaires obtenus en collaboration avec le groupe de Mme L. Leinwand (Albert Einstein College of Medicine, New York) indiquent que, chez l'homme aussi, les taux d'ARNm $\alpha \mathrm{MHC}$ et $\beta \mathrm{MHC}$ varient dans le ventricule et l'oreillette hypertrophiés de même façon que leurs protéines respectives. Cela suggère que la réponse génique est contrôlée en amont de la traduction des ARNm. Des expériences de transcription in vitro à partir de noyaux isolés sont en cours dans notre laboratoire afin de déterminer si la régulation s'effectue au niveau de la transcription des gènes ou au niveau de la stabilité des ARNm.

Les conséquences mécaniques et énergétiques de la transition $\alpha \mathrm{MHC}$ $\rightarrow \beta \mathrm{MHC}$ apparaissent clairement. En effet, la vitesse de raccourcissement d'un muscle dépend, pour un degré d'activation donné, de l'activité ATPasique de l'isomyosine qu'il contient. L'activité ATPasique de l'isomyosine $\mathrm{V} 1$ ( $\alpha \mathrm{MHC}, \alpha \mathrm{MHC})$ est environ quatre fois supérieure à celle de l'isomyosine V3 $(\beta \mathrm{MHC}$, $\beta \mathrm{MHC})$, et une relation significative a été trouvée entre la vitesse de contraction de divers muscles papillaires de rat et de lapin et la proportion de leurs isomyosines, et ce à tous les niveaux de charge relative $[9,10]$. La vitesse de contraction plus lente résultant de la présence de $\beta \mathrm{MHC}$ étant associée à une chaleur de tension titative study. J Am Coll Cardiol 1987; 9 1024-30

8. Izumo S, Lompré AM, Matsuoka R, et al. Myosin heavy chain messenger RNA and protein isoform transitions during cardiac hypertrophy. Interaction between hemodynamic and thyroid-hormone induced signals. J Clin Invest $1987 ; 79$ : 970-7.

9. Schwartz K, Lecarpentier Y, Martin JL et al. Myosin isoenzyme distribution correlates with speed of myocardial contraction. J Mol Cell Cardiol 1981; 13 : 1071-5.

10. Pagani ED, Julian FJ. Rabbit papillary muscle myosin isozymes and the velocity of muscle contraction. Circ Res 1984; 54 : 586-94.

11. Alpert NR, Mulieri LA. Increased myothermal economy of isometric force generation in compensated cardiac hypertrophy induced by pulmonary artery constriction in the rabbit. Circ Res 1982 ; 50 : 940-6.

\begin{tabular}{|c|c|c|c|c|}
\hline \multicolumn{5}{|c|}{$\begin{array}{l}\text { Tableau II } \\
\text { AÎNES LOURDES DE } \\
\text { AQUE NORMAL ET }\end{array}$} \\
\hline & \multicolumn{2}{|c|}{ Homme } & \multicolumn{2}{|c|}{ Rat } \\
\hline & Oreillettes & Ventricules & Oreillettes & Ventricules \\
\hline \multicolumn{5}{|l|}{$\begin{array}{l}\text { Développement } \\
\text { ontogénique }\end{array}$} \\
\hline Fœtus & $\alpha$ & $\beta$ & $\alpha$ & $\beta$ \\
\hline Nouveau-né & $\alpha \beta$ & $\beta_{\alpha}$ & $\alpha \beta$ & $\alpha$ \\
\hline Adulte & $\alpha \beta$ & $\beta_{\alpha}$ & $\alpha \beta$ & $\alpha \beta$ \\
\hline Sénescence & - & $\beta_{\alpha}$ & - & $\alpha \beta$ \\
\hline $\begin{array}{l}\text { Surcharge de travail } \\
\text { hémodynamique }\end{array}$ & $\alpha \beta$ & $\beta$ & - & $\alpha \beta$ \\
\hline
\end{tabular}


plus faible que celle due à $\alpha \mathrm{MHC}[11]$, on admet que la phénoconversion $\alpha \mathrm{MHC} \rightarrow \beta \mathrm{MHC}$ produit une amélioration du rendement du développement de la tension. Il s'agit donc là d'un mécanisme avantageux parce qu'il permet à la fibre cardiaque hypertrophiée de développer à un coût énergétique moindre une tension identique à celle d'une fibre normale.

Isoactines

Deux isoformes sont présentes dans le tissu musculaire cardiaque, l'actine $\alpha$ squelettique ( $\alpha$ sk) et l'actine $\alpha$ cardiaque ( $\alpha$ card), codées par deux gènes situés sur des chromosomes différents $(\alpha$ sk, chromosome 3 ; $\alpha$ card, chromosome 17) [revues dans 5 et 12]. Ce sont des protéines structuralement très proches, ne différant que par 4 acides aminés sur 375. Les ARNm codant pour les deux isoformes sont aussi très proches, composés d'environ 1700 paires de bases, et on ne peut les séparer par simple analyse électrophorétique. Cependant, s'ils comportent des régions d'homologie très fortes (95\% dans la partie 5' codante), ils sont beaucoup plus divergents dans les parties 3' et 5 ' non codantes. Quant aux gènes correspondants, ils comportent chacun 6 introns de taille variable. La localisation des introns dans le gène codant pour l'actine squelettique est identique chez le poulet et le rat, ce qui suggère un gène unique ancestral qui aurait produit par duplication génique les deux isogènes sarcomériques. Les différences de structures entre $\alpha$ sk et $\alpha$ card ont donc été conservées au cours de l'évolution pendant au moins 270 millions d'années. Une surcharge de travail systolique produit dans le cœur de rat une accumulation de l'ARNm $\alpha$ sk, accumulation transitoire et proportionnelle à l'augmentation du poids du cœur [13]. L'actine $\alpha$ sk étant présente dans le ventricule fotal et périnatal [14], cela confirma l'hypothèse d'une réexpression d'un phénotype fœtal dans le cœur adulte hypertrophié (Tableau III). La signification de cette expression transitoire n'est pas connue. In vitro, $\alpha$ sk et $\alpha$ card activent de la même façon l'ATPase de la myosine, mais ce test n'est pas très discriminant. Deux des différences en acides aminés entre $\alpha$ sk et $\alpha$ card sont $m / s n^{\circ} 7$ vol. 6, septembre 90
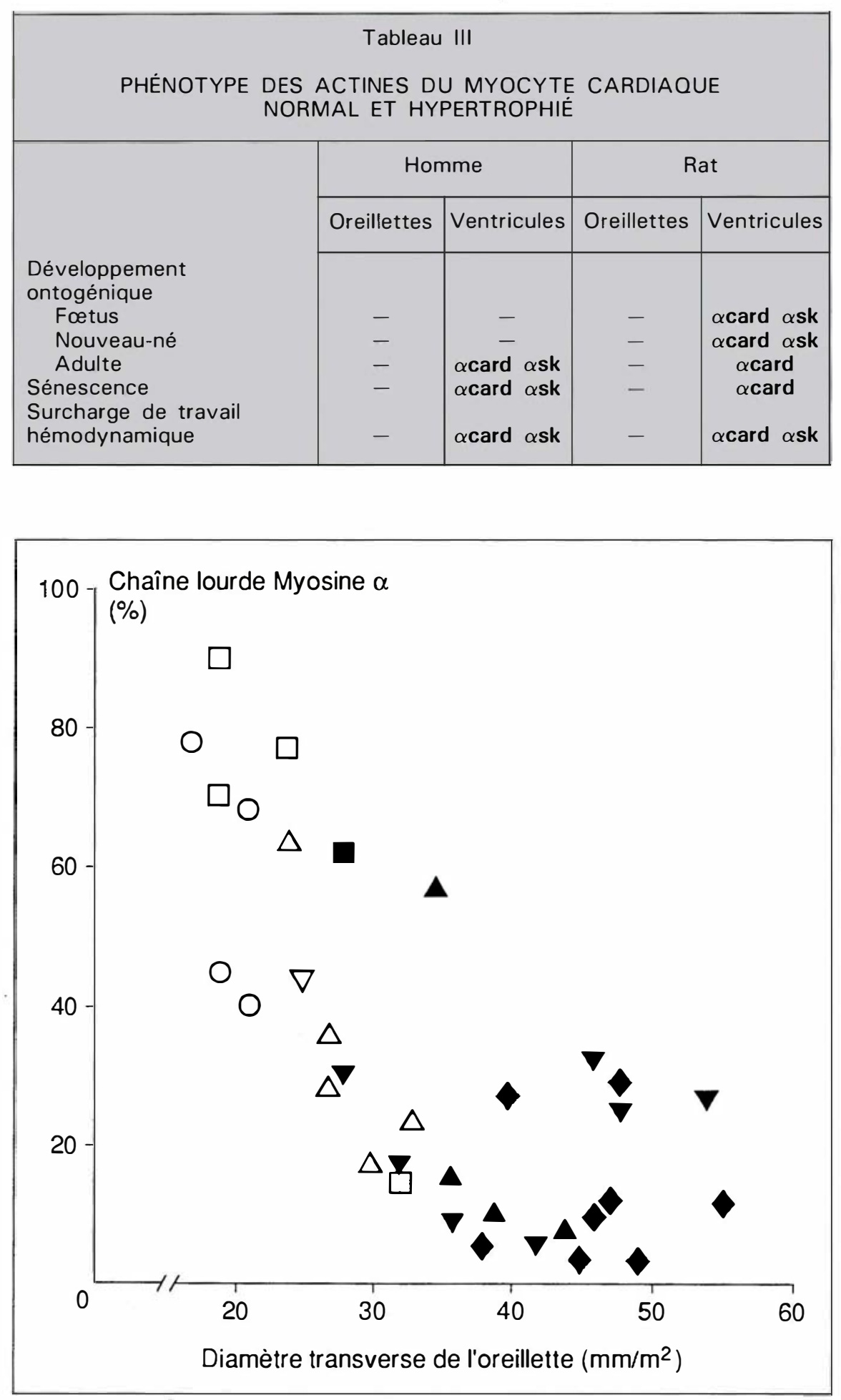

Figure 1. Corrélation entre le diamètre transverse de l'oreillette gauche indexé mesuré par échographie et la proportion de chaîne lourde de type alpha dans la myosine préparée à partir d'une biopsie des oreillettes correspondantes $(n=34, r=0,73, p<0,01)$. majeure ; $\triangle \boldsymbol{\Lambda}$ : rétrécissement mitral pur ; $\nabla \nabla$ : rétrécissement mitral avec petite fuite associée; $\bigcirc$ : syndrome de Wolff-Parkinson-White lvoie anormale de conduction auriculo-ventriculaire). Les symboles blancs représentent les patients en rythme sinusal, les symboles noirs, les patients en fibrillation auriculaire permanente. D'après [7]. 


\section{RÉFÉRENCES}

12. Buckingham $\mathrm{M}$, Alonso $\mathrm{S}$, Barton $\mathrm{P}$, et al. Actin and Myosin multigene families their expression during the formation and maturation of striated muscle. Am J Med Gen 1986 ; 25 : 623-34.

13. Schwartz K, de La Bastie D, Bouveret $\mathrm{P}$, et al. $\alpha$-skeletal muscle actin mRNA's accumulate in hypertrophied adult rat hearts. Circ Res 1986 ; 59 : 551-5.

14. Minty AJ, Alonso S, Caravatti, et al. A fetal skeletal muscle actin mRNA in the mouse and its identity with cardiac actin mRNA. Cell $1983 ; 30$ : 185-92.

15. Reedy MC, Beall C, Fyrberg E. Myosin heads can form reverse rigor chevrons. Nature 1989 ; 339 : 481-3.

16. Garner I, Minty AJ, Alonso S, et al. A 5' duplication of the $\alpha$-cardiac actin gene in $\mathrm{BALB} / \mathrm{c}$ mice is associated with abnormals levels of $\alpha$-cardiac and $\alpha$-skeletal actin mRNAs in adult cardiac tissue. EMBO J 1986 ; 5 : 2559-67.

17. Winegrad S, Wisnewsky C, Schwartz $\mathrm{K}$. The effect of thyroid hormone on the accumulation of mRNA for alpha skeletal and cardiac actin in hearts from normal and hypophysectomised rats. Proc Natl Acad Sci USA $1990 ; 87$ : 2456-60.

18. Starksen NF, Simpson PC, Bishopric $\mathrm{N}$, et al. Cardiac myocyte hypertrophy is associated with $c-m y c$ proto-oncogene expression. Proc Natl Acad Sci USA 1986; 83 : 8348-50.

19. Boxer LM, Prywes R, Roeder RG, et al. The sarcomeric actin CArG-binding factor is indistinguishable from the $c$-fos serum response factor. Mol Cell Biol 1989; 9 : 515-22.

20. De La Bastie D, Wisnewsky C, Schwartz $\mathrm{K}$, et al. $\left(\mathrm{Ca}^{2}+\mathrm{Mg}^{2+}\right)$-dependent ATPase mRNA from smooth muscle sarcoplasmic reticulum differs from that in cardiac and fast skeletal muscles. FEBS Lett $1988 ; 229$ : 45-8.

21. Nagai R, Zarain-Herzberg A, Brandl $\mathrm{CJ}$, et al. Regulation of myocardial $\mathrm{Ca}^{2+}$-ATPase and phospholamban mRNA expression in response to pressure overload and thyroid hormone. Proc Natl Acad Sci situées au niveau des résidus 2 et 3 du peptide NH2-terminal qui serait impliqué dans la liaison avec la tête de la myosine. Deux substitutions dans ce même peptide, mais aux positions 6 et 7, réalisées par mutagenèse dirigée chez la drosophile, ont complètement désorganisé la structure des sarcomères, probablement en modifiant la polarité des filaments d'actine, et ont aboli le vol [15]. On peut donc supposer que les différences aux positions 2 et 3 pourraient aussi faire jouer à chacune des isoactines sarcomériques un rôle spécifique et optimal dans les muscles où elles sont présentes. Un modèle intéressant à analyser dans cette optique serait la souris $\mathrm{BALB} / \mathrm{c}$ qui est porteuse d'une mutation dans la région promotrice de l'isogène $\alpha$ card et dont le cœur contient à peu près autant des deux isoactines, au contraire de celui de la souche non mutée qui ne contient que $\alpha$ card [16].

Une autre possibilité serait que l'actine $\alpha$ sk pourrait être liée au processus d'activation globale des gènes cardiaques pendant la phase initiale de l'hypertrophie. En effet, il existe deux autres situations où la croissance cardiaque est accompagnée d'une activation du gène $\alpha$ sk. D'une part, sous l'effet de la thyroxine, qui produit une hypertrophie du cœur adulte caractérisée par une accéléra- tion de la contraction due à l'activation du gène $\alpha \mathrm{MHC}$. Saul Winegrad, en année sabbatique dans notre équipe, vient de montrer que l'injection de cette hormone à des rats provoque en 6 heures une accumulation des ARNm actine $\alpha$ sk dans le tissu ventriculaire (figure 2). Cette accumulation est trop rapide pour être due aux effets hémodynamiques secondaires de l'hormone, mais elle est simultanée à ses effets sur la croissance cardiaque [17]. D'autre part, les agonistes $\alpha 1$-adrénergiques qui provoquent la croissance de myocytes néonataux activent, dans ces cellules, la transcription du gène actine $\alpha$ sk en 1 à 2 heures [18]. Il existe dans les régions promotrices du gène de l'actine $\alpha$ sk et de l'oncogène $c$-fos des séquences nucléotidiques homologues capables de fixer un facteur ubiquitaire sérique, le serum response factor et des facteurs musculaires, les muscle actin promoter factors 1 et 2 [19]. C-fos est activé dans le cœur après surcharge de travail hémodynamique et il se pourrait donc que l'activation de l'actine $\alpha$ sk fût parallèle à celle de $c$ fos. C-fos a été impliqué dans certains processus de différenciation cellulaire et les phénoconversions du myocyte cardiaque adulte présentent bien des analogies avec la différenciation. Il doit cependant exister un rôle spécifique de l'actine $\alpha$ sk dans le cœur car

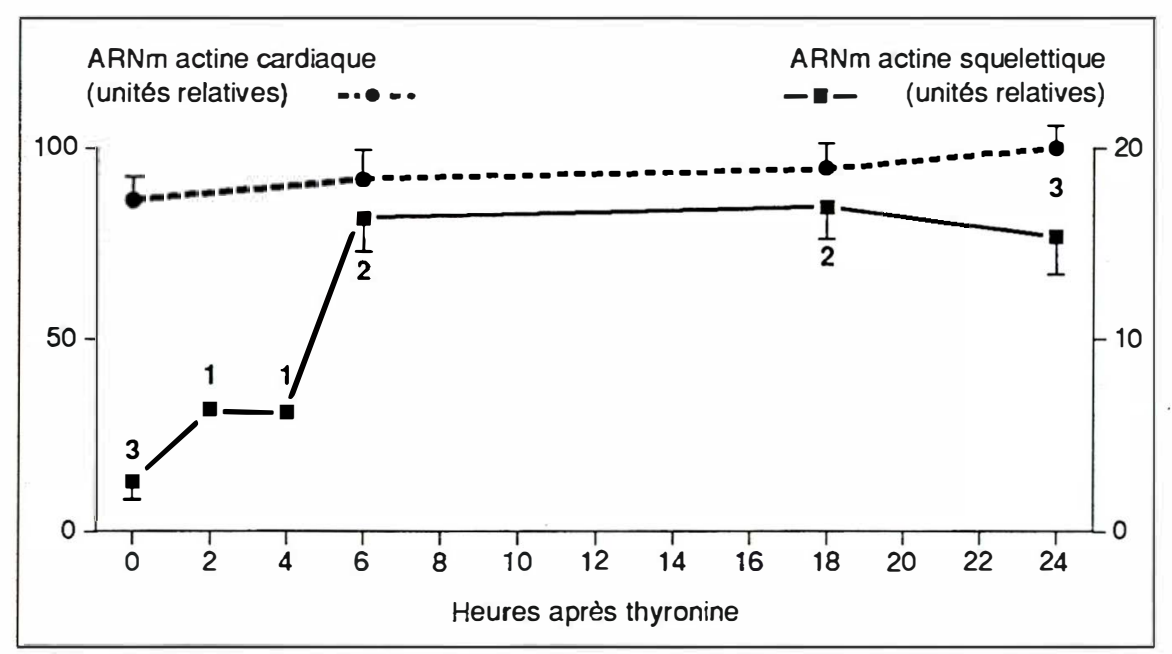

Figure 2. Cinétique de l'accumulation des ARNm codant pour les actines cardiaques et squelettiques dans le cœur de rat après injection intrapéritonéale de thyroxine (5 $\mu \mathrm{g}$ pour $10 \mathrm{~g}$ de poids corporel). Le nombre d'animaux est indiqué à chaque point, et la valeur de chaque animal est la moyenne de 2 à 3 déterminations séparées. D'après [17]. 
1) les facteurs précédents ne se fixent pas sur les gènes $\alpha$ sk et $c-f o s$ avec la même affinité, 2) après sténose aortique, le gène $\alpha$ sk est activé beaucoup plus longtemps que celui de $c$-fos (1 semaine versus 72 heures) et enfin 3) le cœur fœtal, qui ne contient que des traces de $c$-fos, contient beaucoup d'actine $\alpha$ sk. Ce rôle reste à définir.

\section{Non-activation du gène de la calcium-ATPase du réticulum sarcoplasmique}

L'ATPase $\mathrm{Ca}^{++}-\mathrm{Mg}^{++}$dépendante du réticulum sarcoplasmique $\left(\mathrm{Ca}^{++}\right.$-ATPase) permet le transport, contre un gradient de concentration, du calcium libéré par les myofibrilles vers le réticulum sarcoplasmique. A ce titre, cette enzyme est un des principaux responsables de la relaxation de la fibre cardiaque, c'est-à-dire du processus pendant lequel le cœur retourne à sa configuration précontractile. Cette protéine est formée d'une seule chaîne polypeptidique comportant trois domaines cytoplasmiques reliés à dix domaines transmembranaires par un segment formé de cinq hélices sur lesquelles se fixe le $\mathrm{Ca}^{2+}$. Depuis peu l'on sait qu'il s'agit d'une famille multigénique, constituée de deux gènes susceptibles de produire quatre isoformes [revue dans 5]. L'isoforme du cœur adulte est la même que celle des muscles lents du squelette. Le gène correspondant produit par épissage alternatif une isoforme présente dans les muscles lisses et les tissus non musculaires, différant de la précédente par les trois derniers acides aminés et l'addition de quarante-six acides aminés supplémentaires à la partie $\mathrm{C}$ terminale [20].

On pressentait que le recaptage du calcium par le réticulum sarcoplasmique se faisait mal dans l'hypertrophie et l'insuffisance cardiaque. En effet, in vivo, la relaxation cardiaque est ralentie, prolongée, voire incomplète, les mouvements intra-cellulaires de calcium sont prolongés, et la production de chaleur liée à ces mouvements est diminuée. In vitro, le transport de calcium par des vésicules isolées de réticulum sarcoplasmique est perturbé [revue dans 5]. Cependant, les expériences tendant à relier ces $\mathrm{m} / \mathrm{s} n^{\circ} 7$ vol. 6, septembre 90

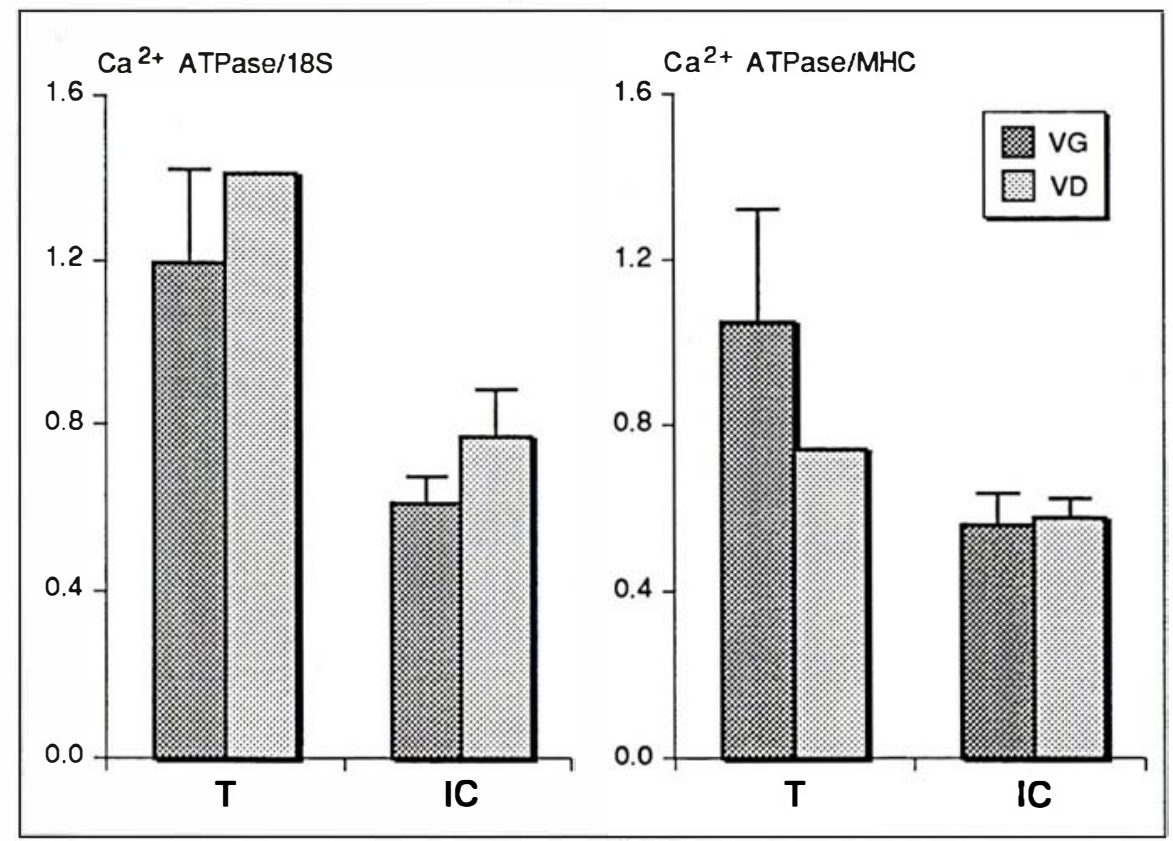

Figure 3. Valeurs moyennes de la proportion d'ARNm codant pour la Ca++-ATPase du réticulum sarcoplasmique de ventricules gauche (VG) et droit (VD) de patients témoins $(+)$ et au stade de l'insuffisance cardiaque terminale (IC). Cette proportion a été déterminée soit par rapport à I'ARN ribosomal $18 S\left(\mathrm{Ca}^{++}\right.$-ATPase/18S), soit par rapport à I'ARNm codant pour la chaîne lourde de la myosine (Ca++-ATPase/MHC). D'après [24].

altérations à celles de la structure de l'enzyme n'avaient pas donné de résultats concluants, essentiellement parce qu'il n'est pas aisé d'analyser une molécule aussi complexe dans un environnement vésiculaire. L'isolement en 1987 de clones spécifiques de l'isoARNm cardiaque permit une autre approche, l'analyse de la nature et de la quantité des ARNm présents dans le cœur hypertrophié. Presque simultanément trois équipes, dont la nôtre (A.M. Lompré, D. de la Bastie), viennent de décrire une diminution relative de l'accumulation de l'isoARNm cardiaque sans accumulation d'une autre isoforme, et ce dans deux modèles animaux différents [21-23]. Cette diminution est corrélée au taux d'enzyme tissulaire indiquant que la régulation du gène est probablement pré-traductionnelle. Elle est également corrélée à la quantité de calcium captée par le réticulum sarcoplasmique, ce qui suggère que l'altération in vitro de la fonction du réticulum est due à une diminution du nombre de molécules de $\mathrm{Ca}^{++}$-ATPase. Par ailleurs, nous avons analysé des échantillons de cœurs explantés provenant de patients au stade d'insuffisance cardiaque terminale et d'étiologies diverses (myocardiopathies dilatées, insuffisants coronaires, régurgitation mitrale). La proportion de l'ARNm est également diminuée, qu'elle soit calculée par rapport à la quantité d'ARN total (ARN 18S) ou par rapport à la quantité de matériel contractile (ARNm codant pour la chaîne lourde de la myosine) et ce quel que soit le type de pathologie (figure 3) [24].

Ainsi il apparaît que le gène de la $\mathrm{Ca}^{++}$-ATPase du réticulum sarcoplasmique n'est pas activé par une surcharge de travail hémodynamique, alors que le cœur in toto grossit. La conséquence en est une densité moindre des molécules d'ATPases dans le cœur hypertrophié. Ce mécanisme expliquerait que le signal aequorine donné par le calcium cytoplasmique soit prolongé dans le cœur hypertrophié puisqu'il y aurait moins d'enzyme capable de faire entrer cet ion dans le réticulum sarcoplasmique et la relaxation s'en trouverait donc prolongée d'autant. On peut souli- 


\section{RÉFÉRENCES}

22. Komuro I, Kurabayashi M, Shibazaki $\mathrm{Y}$, et al. Molecular cloning and characterization of a $\mathrm{Ca}^{2+}+\mathrm{Mg}^{2+}$-dependent adenosine triphosphatase from rat cardiac sarcoplasmic reticulum. Regulation of its expression by pressure overload and developmental stage. J Clin Invest 1989 ; 83 : 1102-8.

23. De La Bastie D, Levitsky D, Rappaport L, et al. Function of the sarcoplasmic reticulum and expression of its $\mathrm{Ca}^{2+}$-ATPase gene in pressure overloadinduced cardiac hypertrophy in the rat. Circ Res 1990 ; 66 : 554-64.

24. Mercadier JJ, Lompré AM, Duc P, et al. Altered sarcoplasmic reticulum $\mathrm{Ca}^{2+-A T P a s e}$ gene expression in the human ventricle during end-stage heart failure. J Clin Invest 1990; 85 : 305-9.

25. Bodak A, Cluzeaud F, Gastineau P, et al. Degree of granulation of atrial cardiocytes : its decrease after aorto-caval fistula in the rat. Basic Res Cardiol 1975 ; 36 : 719-26.

26. Mercadier JJ, Michel JB. Modifications hormonales dans l'hypertrophie et l'insuffisance cardiaque. In : Swynghedauw B, ed. Hypertrophie et insuffisance cardiaque. Paris : John Libbey Eurotext, 1990 : 523-53.

27. Lattion AL, Michel JB, Arnauld E, et al. Myocardial recruitment of ANF mRNA increase with volume overload in the rat. Am J Physiol 1986 ; 251 : H890-6.

28. Yasue H, Obata K, Okumara K, et al. Increased secretion of atrial natriuretic polypeptide (ANP) from the left ventricle in patients with dilated cardiomyopathy. Circulation 1988 ; 78 (supp II) : 586

29. Mercadier JJ, Samuel JL, Michel JB, et al.A trial natriuretic factor gene expression in rat ventricle during experimental hyperin rat ventricle during experimental hyper-

30. Mercadier JJ, Zongazo MA, Wisnewsky C, et al. Atrial natriuretic factor messenger ribonucleic acid and peptide in the human heart during ontogenic development. Biochem Biophys Res Comm 1990 ; 159 . 777-82

31. Kira Y, Kochel PJ, Gordon EE, et al. Aortic pressure as a determinant of cardiac protein synthesis. Am J Physiol $1975 ; 229$ : gner qu'il s'agit là d'un des premiers indices moléculaires de l'insuffisance ventriculaire gauche humaine superposable aux modèles animaux.

\section{Suractivation ventriculaire du gène du facteur atrial natriurétique}

La présence de granules typiques d'une sécrétion protéique dans les cardiocytes auriculaires a valu au peptide effecteur du système le qualificatif d'atrial et il n'est pas inutile de rappeler ici que c'est le groupe de P.Y. Hatt à Paris qui démontra en 1976 que ce système était contrôlé par des modifications du régime hydrosodé ou de la volémie [25]. Le facteur atrial natriurétique est le produit d'un gène unique situé sur le bras court du chromosome 1 chez l'homme. L'ARNm correspondant est traduit en une pré-pro-horrnone de 151 acides aminés, elle-même clivée en un pro-peptide de 126 acides aminés $\gamma$-FAN, qui est la forme de stockage de l'hormone contenue dans les granules auriculaires. La forme active circulante, $\alpha \mathrm{FAN}$, correspond aux 28 acides aminés de l'extrémité $\mathrm{C}$ terminale [revue dans 26].

Les taux circulants de facteur atrial natriurétique sont augmentés jusqu'à 20 à 30 fois dans l'hypertrophie et l'insuffisance cardiaque et l'on pensait que le peptide en excès provenait des seules oreillettes. Il est maintenant tout à fait clair que, si dans le ventricule normal de mammifere adulte le facteur atrial natriurétique et son $\mathrm{ARNm}$ sont à peine détectables, il en est tout autrement au cours de l'hypertrophie du cœur adulte [27]. Dans tous les modèles animaux d'hypertrophie ventriculaire droite ou gauche, dans l'insuffisance cardiaque du hamster syrien cardiomyopathique et dans les ventricules de certains patients au stade d'insuffisance terminale (J.-J. Mercadier, résultats non publiés) les taux de l'ARNm et du peptide immunoréactif sont élevés. La preuve de la participation ventriculaire au taux circulant du facteur atrial natriurétique vient récemment d'être apportée chez l'homme car le peptide a été détecté en quantités importantes dans le sang du sinus coronaire [28]. Nous avions observé chez le rat que le taux ventriculaire d'ARNm pouvait atteindre plus du dixième de celui des oreillettes [29]. La masse ventriculaire étant environ 10 fois supérieure à la masse auriculaire, on peut donc supposer que le ventricule hypertrophié participe autant que l'oreillette à l'augmentation du facteur atrial natriurétique plasmatique.

Les propriétés diurétiques, natriurétiques et vasorelaxantes du facteur atrial natriurétique sont bien connues, de même que son action inhibitrice sur la sécrétion d'aldostérone et de rénine. A la phase d'hypertrophie compensée, la production accrue de facteur atrial natriurétique devrait tendre à diminuer les conditions de charge des fibres cardiaques et ceci constituerait donc un mécanisme avantageux permettant à la fibre de travailler moins. Le gène du facteur atrial natriurétique est actif dans les ventricules embryonnaires et fœtaux [30]. Il y a donc là encore similitude entre phénotype du cœur fœtal et phénotype du cœur adulte hypertrophié.

\section{Facteurs déclenchants}

Un événement mécanique peut-il modifier l'expression génique d'un myocyte cardiaque (Tableau IV) ? Les conséquences immédiates d'une surcharge hémodynamique sur la fibre cardiaque sont au minimum de trois : étirement dû à un engorgement du lit vasculaire (phénomène connu sous le nom d'effet érectile cardiaque), augmentation de la contrainte pariétale ayant pour conséquence une augmentation de la tension isométrique et enfin déséquilibre thermodynamique conduisant probablement à une légère hypoxie cellulaire. Les effets de l'étirement et de la tension ont été clairement mis en évidence à la fois in vitro sur des préparations de cœur isolé, sur des cultures de myocytes néonataux et sur des myocytes adultes maintenus en survie, et in vivo sur des muscles papillaires désinsérés de la paroi ventriculaire. Les résultats indiquent que l'étirement seul active la synthèse des protéines et des ARN [31], que cet effet est plus important si les cellules cardiaques se contractent [32] et enfin que l'activité mécanique serait nécessaire, voire indispensable, à la 
synthèse de la myosine et de l'actine [33]. La preuve directe de l'effet de l'étirement sur l'activité d'un gène cardiaque a tout récemment été apportée par le groupe de Y. Yazaki au Japon. Ces auteurs ont étiré sur une membrane déformable des myocytes néonataux de rat transfectés avec la région promotrice de $c$ fos liée à une portion du gène de la chloramphénicol-acétyl-transférase et ils ont observé que l'enzyme était produite dans les cellules pour un étirement correspondant à $10 \%$ de la longueur initiale [34].

Quels sont les messagers intracellulaires que met en jeu un étirement de la membrane myocytaire? L'an dernier, trois d'entre eux ont été décrits, activation de la voie AMPcdépendante [35], activation de la voie des inositols phosphates [36], et entrée accrue de sodium dans les myocytes [32]. L'activation de la voie AMPc-dépendante est très rapide car, après 15 secondes d'élévation de la pression de perfusion d'un cœur isolé, les taux tissulaires d'AMPc et de la sous-unité catalytique $\mathrm{C}$ des protéine-kinases AMPc-dépendantes sont augmentés [35]. L'importance de cette voie est attestée par le fait que son inhibition par des agents pharmacologiques divers, et en particulier la méthacholine, bloque l'augmentation de synthèse protéique normalement obtenue dans les mêmes conditions expérimentales. L'hypothèse est que l'étirement activerait de façon directe soit l'adénylate-cyclase, soit une protéine $\mathrm{G}$ sarcolemnale, mais l'on ne sait pas encore précisément comment par cette voie seraient initiées les synthèses des ARN et des protéines. La mise en jeu de la voie des inositol-phosphates a été démontrée après 10 minutes d'étirement produit en gonflant un ballon de latex dans le ventricule d'un cœur isolé perfusé [36]. La suite des événements biochimiques n'est pas connue, mais l'on sait: (1) que dans d'autres systèmes cellulaires les inositolphosphates sont impliqués dans la croissance et la mitose cellulaire, et (2) que dans des myocytes néonataux, les agonistes $\alpha 1$ adrénergiques provoquent à la fois hypertrophie cellulaire et augmentation de la transcription du gène actine $\alpha$ sk [37]. $\mathrm{m} / \mathrm{s} n^{\circ} 7$ vol. 6 , septembre 90

Tableau IV

\section{REPRÉSENTATION SCHÉMATIQUE DE LA TRANSDUCTION MÉCANOGÉNIQUE}

Surcharge de travail hémodynamique

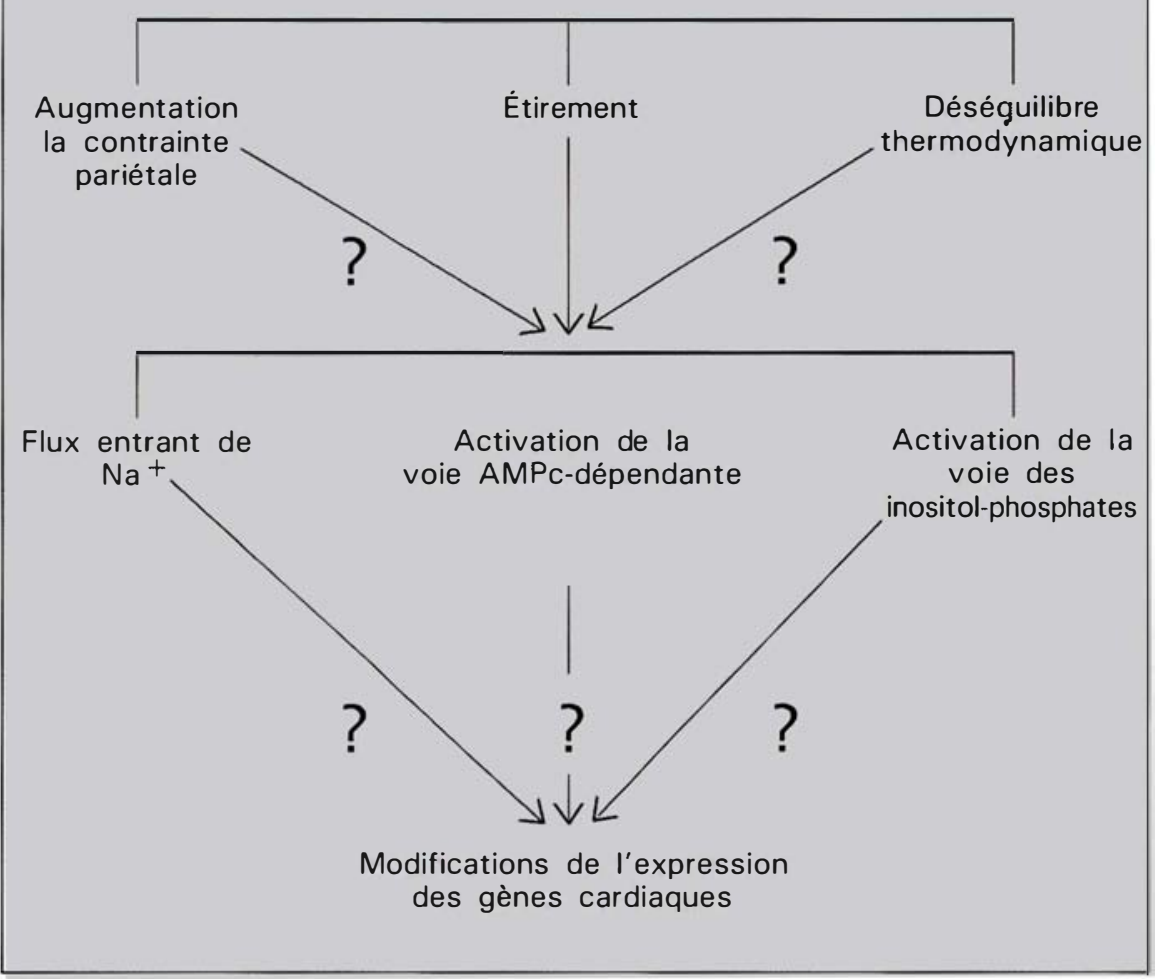

L'hypothèse des auteurs est que l'activation de la protéine kinase C par l'intermédiaire de la phospholipase C entraînerait la phosphorylation d'un facteur de transcription qui se fixerait sur une séquence activatrice du gène actine $\alpha$ sk, dite $\alpha 1$ response element. Ceci reste à prouver. Quant à l'entrée accrue de sodium, elle a été démontrée sur des préparations de muscles papillaires de furet étirés à des longueurs variables, et elle serait due à l'ouverture de canaux ioniques activés par l'étirement (strech-activated ion channels). On sait que dans d'autres tissus leur mise en jeu est très rapide et directe, ne dépendant ni d'une régulation enzymatique ni de la mise en jeu de seconds messagers intracellulaires. Le mécanisme proposé par les auteurs est que l'élévation du sodium intracellulaire activerait l'ATPase sodiumpotassium dépendante, entraînant une élévation du potassium intracellulaire, condition nécessaire à la synthèse des protéines.

Il est tout à fait probable qu'au stimulus purement mécanique s'ajoutent in vivo plusieurs facteurs hormonaux ou peptidiques. Nous avons déjà mentionné l'effet des catécholamines et il est possible, mais non prouvé, que le système nerveux sympathique soit d'une façon ou d'une autre activé in vivo pendant la phase initiale des surcharges de travail hémodynamique. Par ailleurs, l'équipe de $M$. Schneider vient de montrer que les facteurs de croissance TGF $\beta 1$ et $\alpha$ FGF activaient dans des myocytes isolés les gènes $\beta \mathrm{MHC}$ et actine $\alpha$ sk et donc produisaient un phénotype proche de celui des myocytes hypertrophiés [38]. Or ces facteurs sont présents in vivo en quantités importantes dans l'hypertrophie compensatrice qui suit un infarctus expérimen- 


\section{RÉFÉRENCES \\ 32. Kent RL, Hoober JK, Cooper WG Load responsiveness of protein synthesis in adult mammalian myocardium : role of car- diac deformation linked to sodium influx Circ Res $1989 ; 64:$ 74-85 tes $\alpha 1$ adrénergiques [18, 39, 40]. Aucune donnée précise ne permet cependant d'établir une relation de cause à effet entre l'activation de ces oncogènes et celles des autres gènes cardiaques.}

33. Dubus I, Samuel JL, Marotte F, et al. $\beta$-adrenergic agonists stimulate the synthesis of noncontractile but not contractile proteins in cultured myocytes isolated from adult rat heart. Circ Res 1990 ; 66 : 867-74.

34. Komuro I, Kaida T, Shibazaki Y, et al. Stretching cardiac myocytes stimulates protooncogene expression. J Biol Chem $1990 ; 265$ : 3595-8

35. Xenophontos X, Watson PA, Chua $\mathrm{BHL}$, et al. Increased cyclic AMP content accelerates protein synthesis in rat heart. Circ Res 1989 ; 65 : 647-56.

36. Von Harsdorf R, Lang RE, Fullerton M, et al. Myocardial stretch stimulates phosphatidylinositol turnover. Circ Res 1989 65 : 494-501.

37. Long CS, Ordahl CP, Simpson PC $\alpha 1$-adrenergic receptor stimulation of sarcomeric actin isogene transcription in hypertrophy of cultured rat heart muscle cells. $J$ Clin Invest $1989 ; 83$ : 1078-82.

38. Parker TG, Packer SE, Schneider MD. Peptide growth factors can provoke "fetal " contractile protein gene expression in rat cardiac myocytes. I Clin Invest 1990 ; 85 507-14.

39. Bauters C, Moalic JM, Bercovici J, $e$ al. Coronary flow as a determinant of $c-m y c$ and $c$-fos proto-oncogene expression in an isolated adult rat heart. $J \mathrm{Mol}$ Cell Cardio 1988 ; 20 : 97-101.

40. Komuro I, Kurabayashi M, Takaku F, et al. Expression of cellular oncogenes in the myocardium during the developmental stage and pressure-overloaded hypertrophy in the rat heart. Circ Res 1988 ; 62 : 1075-9.

41. Schiaffino S, Samuel JL, Sassoon D, et al. Non-synchronous accumulation of $\alpha$ skeletal actin and $\beta$-myosin heavy chain mRNAs during early stages of pressure overload-induced cardiac hypertrophy demonstrated by in situ hybridization. Circ tal et on peut donc imaginer que, produits par les cellules cardiaques non musculaires, ils seraient un des maillons de la transduction mécanogénique. Enfin, les proto-oncogènes $c$-myc et $c$-fos, qui codent pour des facteurs de transcription, sont activés dans le cœur de façon transitoire dès la première heure qui suit une surcharge de travail hémodynamique, et $c-m y c$ est également activé dans des myocytes néonataux par les agonis-

\section{Régulation coordonnée ou pas?}

Chaque fibre est soumise à une tension différente du fait de la géométrie des cavités cardiaques et, jusqu'à il y a environ 2 à 3 ans, l'on pensait que la tension supportée par cha- que fibre conditionnait sa réponse génique et donc que tous les changements produits par les surcharges de travail hémodynamique devaient se produire au même moment dans les mêmes myocytes. Or il n'en est rien. Nous avons en effet observé par hybridation in situ qu'à la $4^{\mathrm{e}}$ heure qui suit une sténose de l'aorte thoracique, les $\mathrm{ARNm}$ actine $\alpha$ sk sont répartis de façon diffuse dans l'ensemble de la paroi ventriculaire gauche alors que les ARNm $\beta \mathrm{MHC}$ n'apparaissent qu'après 24-48 heures et seulement dans les régions sousendocardiaques et autour des grosses artères coronaires (figure 4) [41]. Ce n'est qu'après une semaine environ que les $\mathrm{ARNm} \beta \mathrm{MHC}$ sont présents dans tout l'ensemble de la paroi ventriculaire gauche. Le gène $\beta \mathrm{MHC}$ est environ six fois plus long que celui de l'actine $\alpha$ sk. L'accumulation plus tardive des ARNm $\beta \mathrm{MHC}$ pourrait donc s'expliquer simplement par le fait qu'après un stimulus unique, il faut plus de temps pour transcrire le

\section{$\beta \mathrm{MHC}$}

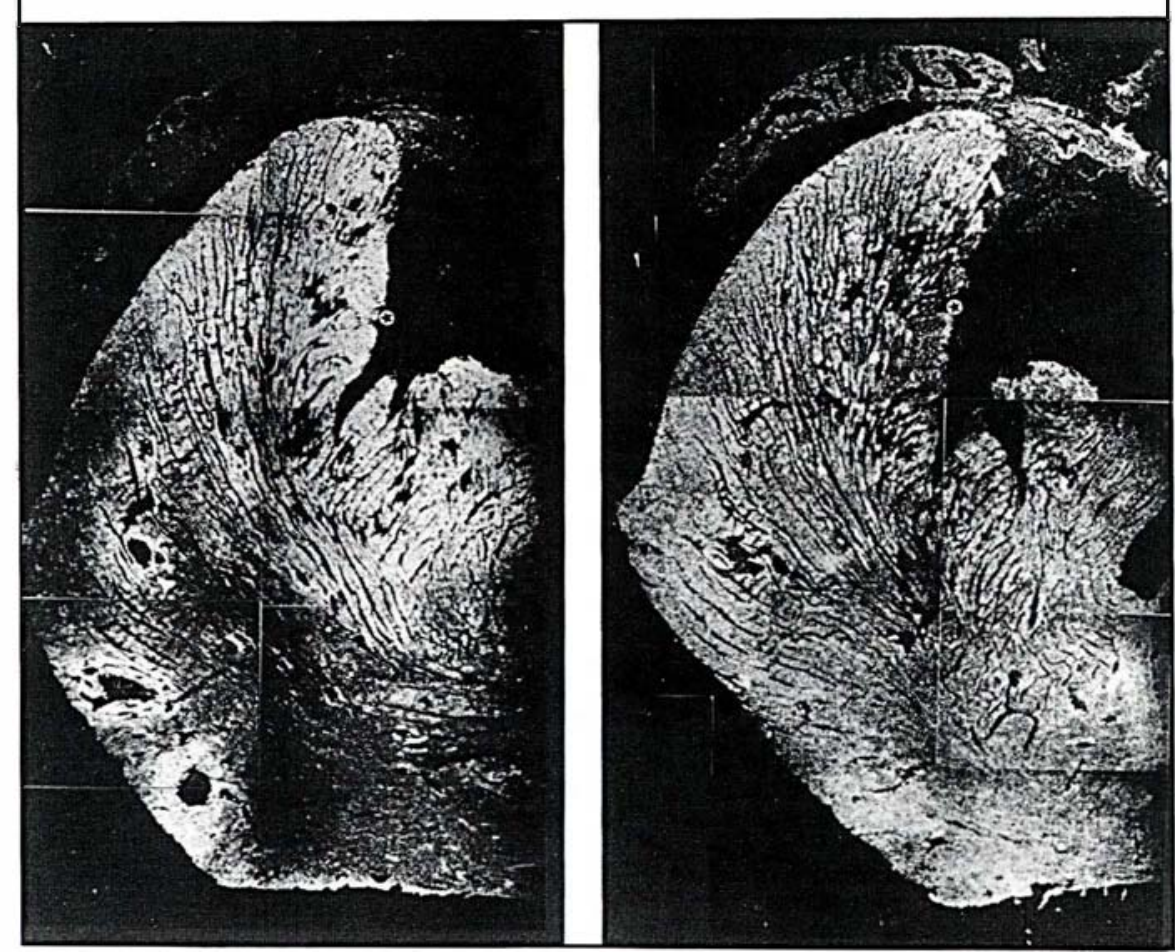

Figure 4. Analyse par hybridation in situ de la répartition tissulaire des ARNm codant pour la chaîne lourde $\beta$ de la myosine ( $\beta$ MHC) et pour I'actine $\alpha$-squelettique ( $\alpha$ skactine) dans le cœur de rat, quarante-huit heures après une sténose de I'aorte thoracique. Reproduit de [41]. Photographies : J.L. Samuel et S. Schiaffino. 
gène correspondant. Cependant, la localisation différente des ARNm $\beta \mathrm{MHC}$ et $\alpha$ sk suggère que plusieurs mécanismes sont mis en jeu. Les $\mathrm{ARNm} \beta \mathrm{MHC}$ s'accumulent dans les régions où soit la tension (sousendocarde), soit l'étirement (autour des artères) sont plus grands. Cela n'est pas le cas pour l'ARNm $\alpha$ sk, et il semble donc établi que les familles des deux principales protéines contractiles ne suivent pas un schéma coordonné de régulation génique au cours de la croissance qui accompagne les surcharges de travail hémodynamique.

\section{Conclusions}

Les surcharges hémodynamiques sont caractérisées par une transduction mécanogénique qui modifie le phénotype cardiaque afin, vraisemblablement, d'adapter celui-ci à de nouvelles conditions de fonctionnement. L'hypertrophie tend à normaliser la contrainte pariétale, la production accrue de facteur atrial natriurétique diminue les conditions de charge et ainsi seraient améliorées les conditions du travail cardiaque. Ce que l'on sait de la signification de l'expression différentielle des familles multigéniques de la myosine et peut-être aussi de la créatine phosphokinase (augmentation de la forme B aux dépens de la forme M) permettrait au cœur de se contracter plus efficacement. Quant à la relaxation ralentie, elle serait aussi plus économique pour la cellule et permettrait d'ajuster la relaxation aux nouvelles conditions de la contraction. Les mécanismes impliqués, modulations de gènes uniques ou expression différentielle de familles multigéniques, sont les mécanismes normalement utilisés au cours de la croissance et de la différenciation musculaire. Presque toutes les protéines, et en particulier les récepteurs et les canaux ioniques, sont membres de familles multigéniques et des données encore fragmentaires montrent que leur expression change aussi dans le cœur hypertrophié. Rien n'empêche donc de penser que de nouvelles voies thérapeutiques seront un jour ouvertes, consistant à stimuler la synthèse d'autres isoformes dans le myocarde ou les vaisseaux, prévenant ainsi ou tout du moins retardant l'apparition de l'insuffisance cardiaque

$\mathrm{m} / \mathrm{s} n^{\circ} 7 \mathrm{vol} .6$, septembre 90

\section{Summary}

Phenoconversion and mechanogenic transduction of the mammalian heart

Chronic hemodynamic overload produces cardiac hypertrophy, and the systolic and diastolic properties as well as the endocrine function of this organ are altered. The genetic mechanisms involved in these alterations are beginning to be understood : 1) the phenotype of contractile proteins is modified by differential expression of myosin and actin multigene families, 2) the density of the key-enzyme of relaxation, the sarcoplamsic reticulum $\mathrm{Ca}^{++}-\mathrm{ATPase}$, is decreased due to a non-activation of the corresponding gene and finally 3 ) there is an increased production of atrial natriuretic factor due to the ventricular activation of the gene. This provides new insights into the understanding of the physiopathology of left ventricular hypertrophy and heart failure, the main cause of morbidity and mortality in our countries.

\section{Erratum}

Dans l'article de J.P. Revillard $\left(\mathrm{m} / \mathrm{s} n^{\circ}\right.$ spécial, août 1990 page 36, lignes 17-35) il fallait lire :

En fonction de cette règle générale, il n'est pas surprenant que les récepteurs de certains agents infectieux soient précisément des molécules de membrane cellulaire ayant la fonction de récepteurs vis-à-vis d'autres molécules de l'organisme : CD4, récepteur du HIV, se lie aux antigènes de classe II du $\mathrm{CMH}$ [33] ; ICAM-1, récepteur des rhinovirus et de certains virus coxsakie $[34,35]$ se lie à LFA-1 (CD11-a); CR2 (CD21) récepteur du virus $\mathrm{EB}$ fixe le fragment C3d.g du complément ; CR3 (CD11b), récepteur du fragment $\mathrm{C} 3 \mathrm{~b}$ du complément est aussi le site de liaison des lipopolysaccharides des bactéries gram-[36] et de certains polysaccharides de levures ou de parasites (Tableau l).

D'autre part, page $34,2^{\text {e }}$ paragraphe, ligne 12, lire CD 45R + au lieu de CD $45 \mathrm{R}^{-}$.

\section{TIRÉS A PART}

K. Schwartz. 